Supporting Information

\title{
The Synergistic Effect of Surface Chemistry and Surface Topography Gradient on Osteogenic/Adipogenic Differentiation of hMSCs
}

Xujie Liu ${ }^{a}$, Yakun Wang, Yan He ${ }^{a, *}$, Xiaofeng Wang ${ }^{b *}$, Ranran Zhang ${ }^{c}$, Akash Bachhuka $^{d}$, Rahul Madathiparambil Visalakshan ${ }^{d}$, Qingling Feng ${ }^{c}$, and Krasimir Vasilev $^{d}$

a School of Biomedical and Pharmaceutical Sciences, Guangdong University of Technology, Guangzhou 510006, China

b Department of Hand surgery, Ningbo No. 6 Hospital, Ningbo, Zhejiang, China. 315040

c State Key Laboratory of New Ceramics and Fine Processing, School of Materials Science and Engineering, Tsinghua University, Beijing 100084, China d Unit of STEM, University of South Australia, Mawson Lakes 5095, Australia

*Corresponding authors:

Yan He: E-mail: heyan129@gdut.edu.cn

Xiaofeng Wang: E-mail: nblywxf@126.com 


\section{Experimental:}

\section{Attenuated total reflectance-Fourier transform infrared spectroscopy (ATR-}

FTIR): ATR-FTIR (ATR Spectrum two, PerkinElmer) was used to characterize the surface chemistry fabricated from plasma polymerized octadiene and allylamine in a wavelength range of $4000-450 \mathrm{~cm}^{-1}$. Potassium Bromide $(\mathrm{KBr})$ powder was used as background whereas the plasma coated $\mathrm{KBr}$ powder was used to characterize surface chemistry. FTIR spectrum was obtained by performing 16 scans over a resolution of $4.0 \mathrm{~cm}^{-1}$. All measurements were carried out in transmittance mode.

\section{Results:}

ATR- FTIR was employed to further access the changes in surface chemistry fabricated using plasma polymerized octadiene and allylamine. In plasma polymerized octadiene, asymmetric $\mathrm{C}-\mathrm{H}$ stretch of methylene and methyl groups were observed at 2917 and $2958 \mathrm{~cm}^{-1}$ respectively (Figure S1a). This demonstrates that the coating is formed from organic compound containing at least one aliphatic fragment or center. O$\mathrm{H}$ stretching was observed at $3393 \mathrm{~cm}^{-1}$ due to the hydration of the samples. The $\mathrm{C}=\mathrm{C}$ stretching was observed at $1554 \mathrm{~cm}^{-1}$ corresponding to unsaturated hydrocarbon. The $\mathrm{C}-\mathrm{H}$ and the $\mathrm{C}=\mathrm{C}$ bend were observed at 1058 and $1400 \mathrm{~cm}^{-1}$ respectively further indicating the presence of a long chain aliphatic compound. ${ }^{1}$ Whereas in plasma polymerized allylamine (Figure S1b), O-H stretching was observed at $3383 \mathrm{~cm}-1$ due to the hydration of the samples. $\mathrm{N}-\mathrm{H}$ stretching showing the presence of secondary amine or imine was observed between $3200-3400 \mathrm{~cm}^{-1}$. The broader peak is observed due to the breaking and recombination of the bonds in plasma polymers. ${ }^{2} \mathrm{C}-\mathrm{H}$ 
stretching was observed at 2903 and $2974 \mathrm{~cm}^{-1}$ which might be an effect of stretching of the imine group. ${ }^{3} \mathrm{C}=\mathrm{N}$ stretching was observed at $1566 \mathrm{~cm}^{-1}$ which can be due to the bending of amine or the stretching of the imine groups. ${ }^{4} \mathrm{~N}-\mathrm{H}$ bending was observed at $1403 \mathrm{~cm}^{-1}$, whereas C-N stretching were observed at $1058 \mathrm{~cm}^{-1}$ and $1250 \mathrm{~cm}^{-1}$ respectively. These results further validate our XPS data showing the successful modification of surfaces with plasma polymerized octadiene and allylamine chemistry.

\section{Supporting Figures}
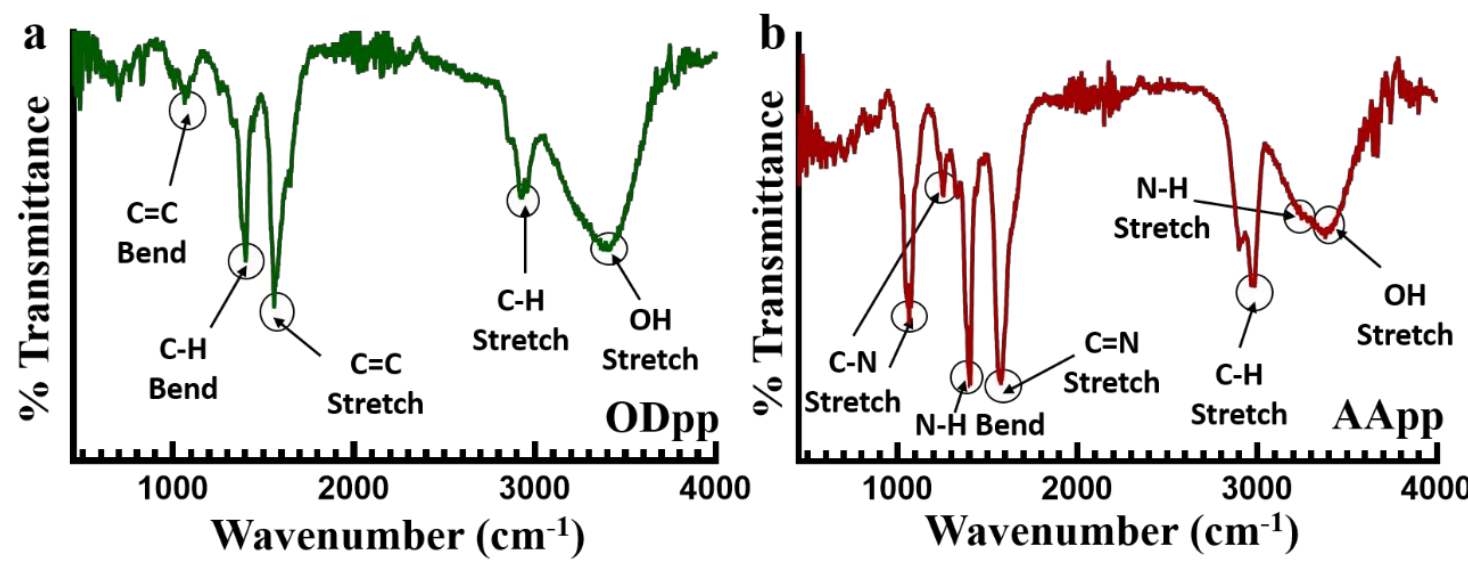

Figure S1. ATR- FTIR spectrum of plasma coated a) octadiene and b) allylamine demonstrating the successful modification of surfaces. 
A) ppAA
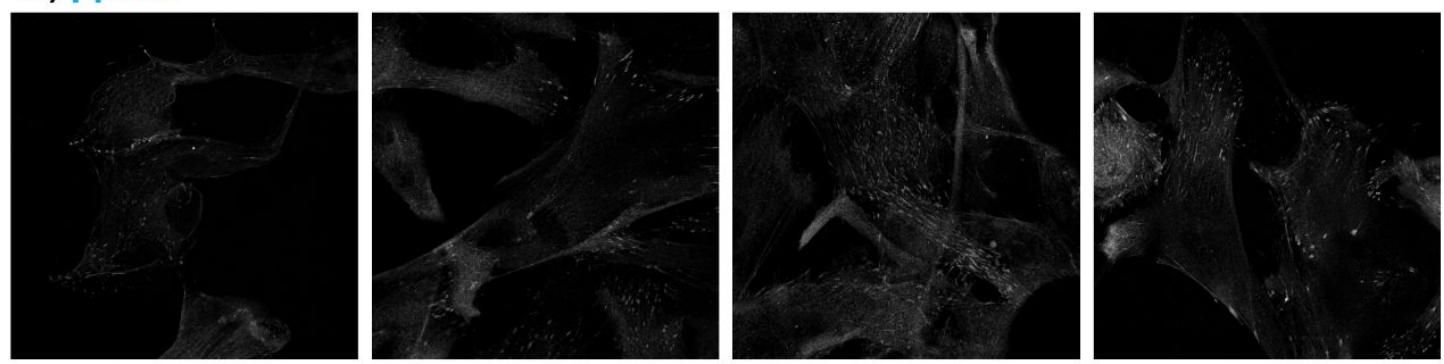

B) $\mathrm{ppOD}$

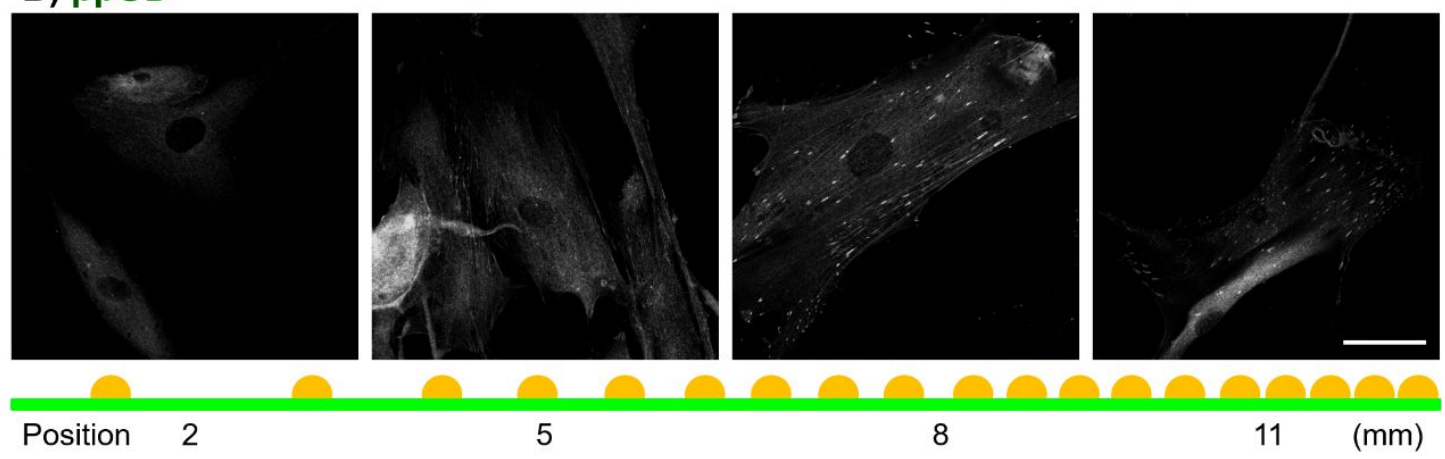

Figure S2 Grayscale images for paxillin staining. Scale bar: $50 \mu \mathrm{m}$

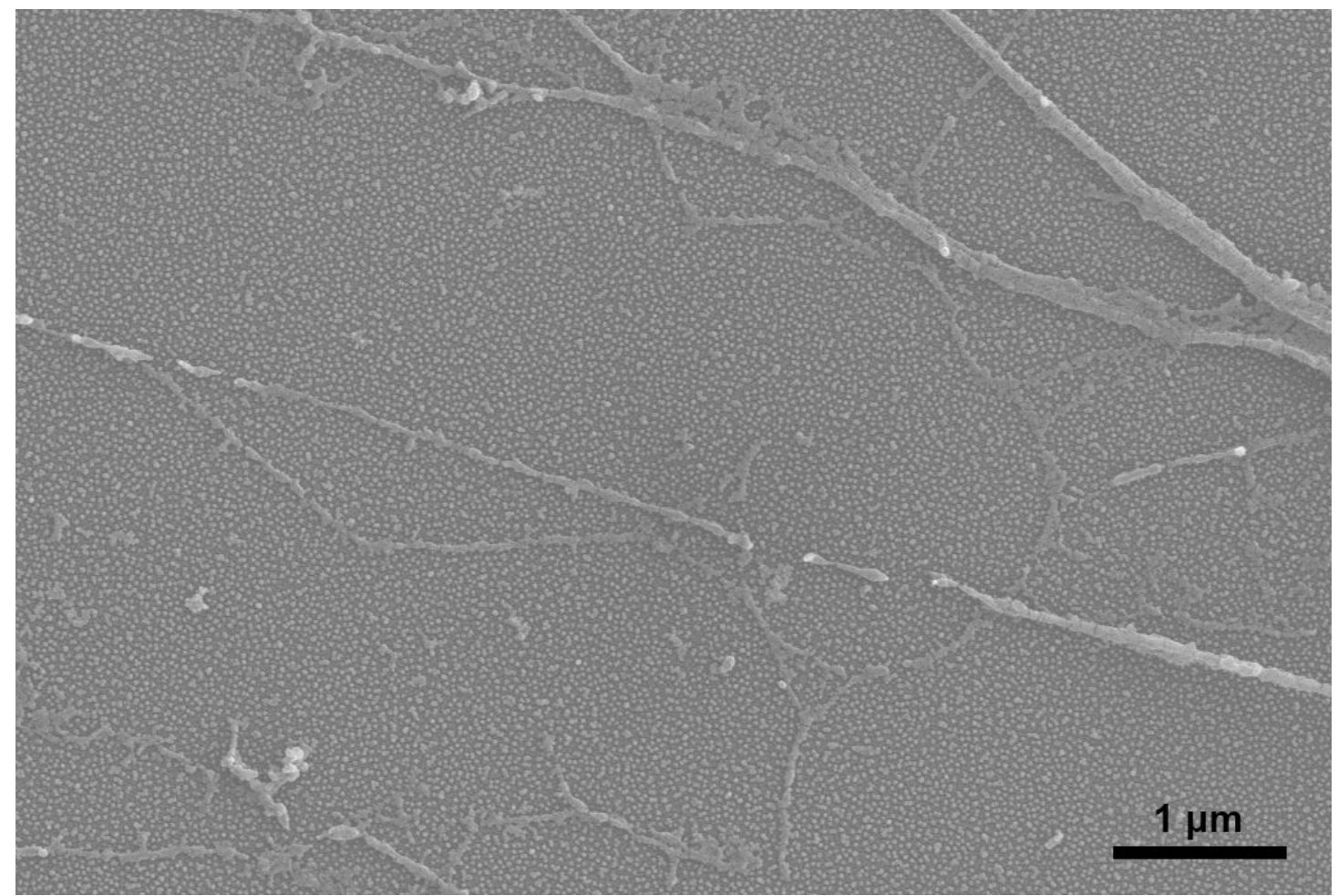

Figure S3 SEM image of hMSCs after culturing for 7 days on the gradient surface. Scale bar: $1 \mu \mathrm{m}$. 


\section{Supporting Tables}

Table S1. p value of a student's t test for hMSCs differentiation responded to different surface chemistry (ppAA and ppOD) for a certain surface topography.

\begin{tabular}{ccc}
\hline & Osteogenesis fraction & Adipogenesis fraction \\
\hline $\mathbf{2} \mathbf{~ m m}$ & 0.000 & 0.176 \\
$\mathbf{5} \mathbf{~} \mathbf{m}$ & 0.005 & 0.001 \\
$\mathbf{8} \mathbf{~ m m}$ & 0.549 & 0.037 \\
$\mathbf{1 1} \mathbf{~ m m}$ & 0.456 & 0.013 \\
\hline
\end{tabular}


Table S2. p value of a two-way ANOVA for hMSCs differentiation responded to different surface topography gradient and surface chemistry.

\begin{tabular}{ccc}
\hline & Osteogenesis fraction & Adipogenesis fraction \\
\hline Topography* & 0.000 & 0.000 \\
Chemistry $^{\#}$ & 0.000 & 0.000 \\
Topography $\times$ Chemistry & 0.000 & 0.139 \\
\hline
\end{tabular}

* Topography: different position on the gradient surface, i.e. 2, 5, 8, and $11 \mathrm{~mm}$

\# Chemistry: ppAA or ppOD 
Table S3. p value of post hoc comparisons with LSD method for cell number responded to different surface topography gradient.

\begin{tabular}{lccc}
\hline & Osteogenesis fraction & Adipogenesis fraction \\
\hline $\mathbf{2} \mathbf{~ m m}$ & $\mathbf{5} \mathbf{~} \mathbf{m}$ & 0.004 & 0.002 \\
& $\mathbf{8} \mathbf{~} \mathbf{m}$ & 0.000 & 0.000 \\
& $\mathbf{1 1} \mathbf{~ m m}$ & 0.000 & 0.000 \\
$\mathbf{5} \mathbf{~ m m}$ & $\mathbf{8} \mathbf{~ m m}$ & 0.000 & 0.000 \\
& $\mathbf{1 1} \mathbf{~ m m}$ & 0.000 & 0.000 \\
$\mathbf{8 ~} \mathbf{~ m m}$ & $\mathbf{1 1} \mathbf{~ m m}$ & 0.000 & 0.004 \\
\hline
\end{tabular}

\section{References}

(1) Coates, J. Interpretation of infrared spectra, a practical approach. Encyclopedia of analytical chemistry: applications, theory and instrumentation 2006.

(2) Myung, S. W.; Choi, H. S. Chemical structure and surface morphology of plasma polymerized-allylamine film. Korean Journal of Chemical Engineering 2006, 23 (3), 505-511.

(3) Krishnamurthy, V.; Kamel, I. L.; Wei, Y. Analysis of plasma polymerization of allylamine by FTIR. Journal of Polymer Science Part A: Polymer Chemistry 1989, 27 (4), 1211-1224.

(4) Van Os, M.; Menges, B.; Foerch, R.; Vancso, G. J.; Knoll, W. Characterization of plasmapolymerized allylamine using waveguide mode spectroscopy. Chemistry of materials 1999, 11 (11), 3252-3257. 